\title{
Lifestyle interventions for the treatment of non-alcoholic fatty liver disease
}

\author{
This article was published in the following Dove Press journal: \\ Hepatic Medicine: Evidence and Research \\ 10 December 2013 \\ Number of times this article has been viewed
}

\section{$\checkmark$ Bradford \\ JF Dillon \\ $\mathrm{MH}$ Miller}

Gut Group, Medical Research Institute, University of Dundee, Ninewells Hospital and Medical School, Dundee, Scotland
Correspondence: MH Miller

Gut Group, Medical Research Institute, University of Dundee, Ninewells Hospital and Medical School, Dundee, DDI 9SY, Scotland

Tel +44 I382 660 III
Abstract: The burden of non-alcoholic fatty liver disease (NAFLD) worldwide is a significant clinical and public health issue, affecting approximately one third of the Western population. This review assesses the effect and impact lifestyle interventions have on the treatment of this common condition. We review studies comparing the effect of calorie restriction and exercise programs, as well as comparison of lifestyle intervention with pharmaceutical intervention. Both calorie restriction and exercise programs are shown to be beneficial in improving features of metabolic syndrome and surrogate markers of NAFLD. The paucity of studies using histological improvement hinders the ability to conclude a benefit on improvement of histological NAFLD, although this is shown in a small number of studies. There is a need to extend the intervention period to show a sustained improvement with intervention as most studies have a follow up period of 12 months of less.

Keywords: NAFLD, lifestyle intervention, exercise, diet

\section{Introduction}

Non-alcoholic fatty liver disease (NAFLD) is now regarded as the most common liver condition in the developed world, affecting up $30 \%$ of the population. It is closely linked with metabolic syndrome and its component parts including obesity, type II diabetes mellitus, and dyslipidemia. Numerous pharmacological interventions have been tested for the treatment of NAFLD with varying success. Nonetheless, no current pharmacological therapy is recommended for the treatment of this condition. Much focus has been given to the role of lifestyle intervention in NAFLD, including the role of prescribed exercise regimes and diets. This review aims to summarize the literature surrounding this approach, highlighting the impact and role these strategies have on NAFLD.

\section{Methods}

We searched a public domain database (PubMed) with the following categories: disease (NAFLD, fatty liver, non-alcoholic steatohepatitis [NASH], and simple steatosis) and intervention (lifestyle intervention, diet, exercise, and behavior) with each possible combination. From this list, papers were considered for further review if they were written in English and involved adult patients only.

The heterogeneity of the literature reviewed enabled us to collate studies together under broad headings, dealing with each of the main lifestyle intervention options. 
However, this heterogeneity also highlighted some salient issues, in particular standardization of a NAFLD phenotype. This varies between studies with histological classification considered the gold standard. This classification is not always feasible or possible. Where appropriate we have highlighted the descriptors used in defining the NAFLD phenotype. Furthermore, we have included studies of features of metabolic syndrome, especially where liver health was considered and reviewed.

\section{Results and discussion}

NAFLD is defined as fat accumulation in the liver, greater than $5 \%-10 \%$ by weight, in the absence of excessive alcohol consumption. ${ }^{1}$ It is a spectrum disease, ranging from simple steatosis without inflammation, to NASH, progressing onwards to fibrosis and cirrhosis. ${ }^{2}$ NAFLD is closely associated with obesity and Type II diabetes mellitus (T2DM); indeed, $75 \%$ of patients with NAFLD are obese, and it is frequently seen as the hepatic manifestation of metabolic syndrome. ${ }^{3}$ The pathophysiology of NAFLD is not completely understood, but insulin resistance is thought to play a part with increased lipolysis leading to an influx in free fatty acid into the liver. ${ }^{2}$

The gold standard for diagnosis of NAFLD is liver biopsy; this is the only way of truly differentiating between the different stages of NAFLD. Biopsy is an imperfect gold standard and has issues pertaining to morbidity, mortality, as well as intra and inter observer variability. Ultrasound (US) is used in most cases; the disadvantage of this is that fat is only picked up when it reaches levels of $33 \%{ }^{3}$ and US is a poor detector of inflammation.

NAFLD was relatively unknown prior to the early 1980s, but is fast becoming the most common chronic liver disease worldwide, ${ }^{4}$ with an estimated prevalence of $20 \%-30 \%$ in Western countries. ${ }^{5}$ The British transplant guidance website has also reported that in 2008 and $200912 \%$ of the patients on the waiting list for liver transplantation had NASH cirrhosis. ${ }^{6}$ This increase in cases of NAFLD is strongly linked to the rising rate in obesity; the National Health Service (NHS) database shows the percentage population with obesity has almost doubled in the last 8 years, with now roughly a quarter of the population having a raised body mass index (BMI). Changing lifestyles is ultimately to blame for this, including increased access to cheap food, eating on the go, and marketing of high calorific foods. ${ }^{7}$

Given all the above mentioned, there has been increased interest in NAFLD and possible ways of managing and treating it. There have been many different suggestions for pharmaceuticals that could be used, including metformin, vitamin E, urodeoxycholic acid, to name a few. ${ }^{1}$ As of yet none of these treatments have shown any significant disease improvement and none are currently recommended. This paper reviews lifestyle modifications as a way of improving the outcome of patients with NAFLD.

\section{Diet and exercise focused regimes}

In this section, we describe studies which have assessed a combination of, or a comparison between, diet and exercise based interventions.

In one of the few studies of lifestyle intervention using histological endpoints, Promrat et $\mathrm{al}^{8}$ conducted a small study ( $\mathrm{n}=30$ ) of patients randomly assigned to receive either diet, exercise, and behavioral strategies or a control group (2:1 ratio). The follow up period was 2 years, and liver biopsies were performed pre and post study period. The lifestyle intervention group had significant improvements in histology at 48 weeks, with $67 \%$ improving their NAFLD activity score to $\leq 2$. There was improvement in hepatic steatosis, lobular inflammation, parenchymal inflammation, and ballooning injury in the interventional group; but results showed no improvement in fibrosis in either group. Liver enzyme and weight loss were also significantly improved in the intervention group. One of the interesting observations from this study was the low attrition rate; 28 of the 30 recruits completed the study period, including follow up biopsy. There was a financial incentive to the patient for completion of the study.

Biopsy results were also used in a study by Jin et al. ${ }^{9}$ One hundred and twenty living donors with NAFLD who had undergone a biopsy and were then encouraged to lose $5 \%$ of their body weight, by diet and exercise, were re-biopsied. There were no specific guidelines for how the patients should lose their weight, which was over an average period of 10 weeks. Over this period 92 lost weight (on average $3 \mathrm{~kg}$ ), 15 gained weight and the remainder maintained their weight. One hundred and three biopsies showed improvement, 82 of these were in those who had lost weight. Further analysis also showed that these improvements were greatest in those who were younger and had the largest decrease in BMI and cholesterol. The authors concluded that a target decrease in body weight should be $5 \%$ and decrease in cholesterol should be $10 \%$ in order to achieve a significant improvement in liver health.

Histological end points were also sought in a paper from Bhat et $\mathrm{al}^{10}$ assessing whether lifestyle modifications can improve NAFLD, although they were not able to biopsy 
their entire cohort. Sixty patients were required to carry out 45 minutes of exercise five times a week as well as an energy restricted diet; $60 \%$ carbohydrate, $20 \%$ fat, $20 \%$ protein. At the end of a 6 month period 45 of the 60 were considered compliant of the intervention. Eight patients had repeat liver biopsies in the compliant group and of these, six showed improvement in steatosis and necro-inflammatory score, with no change in fibrosis. The other two showed no improvement in necro-inflammatory score. Unfortunately there are no biopsy results available for the non-compliant group, indeed there is no record if there were any taken. Insulin resistance and liver function tests (LFT)s were also shown to decrease in the compliant group.

Lazo et $\mathrm{al}^{11}$ recruited 96 patients with T2DM and magnetic resonance imaging (MRI) evidence of hepatic steatosis. Patients were allocated to either the intervention group (calorie restriction and exercise) or control, with a target weight loss of $10 \%$ from baseline. Calorie restriction of 1,200-1,500 calories/day for those with a weight of less than $141 \mathrm{~kg}$ and 1,500-1,800 calories/day for those weighing greater then $141 \mathrm{~kg}$ was encouraged. Also, an increase in exercise to 175 minutes of moderate intensity exercise per week was required and participants attended weekly and monthly counseling sessions. Results were not available on how many achieved a $10 \%$ weight loss, but average percentage weight loss for the interventional group was $8.3 \%$ and $0.03 \%$ in the control group. Although no significant difference was observed in liver enzymes, follow up MRI showed a significant reduction in steatosis following intervention, as well as between the intervention and control groups. Follow up MRI scanning was performed after 12 months of intervention.

In a similar study, Shah et $\mathrm{al}^{12}$ assessed the additive effect of an exercise program to calorie restriction, using MRI to assess hepatic fat. Over a 6 month period, patients were either allocated to a calorie restriction alone group, or a calorie restriction plus exercise group. Calorie restriction was of 500-1,000 calories/day with $30 \%$ being fat, $50 \%$ carbohydrates, and $20 \%$ protein. Exercise requirements were 90 minutes three times a week in an indoor facility supervised by a personal trainer. Both groups showed improvements in steatosis (measured by MRI), liver enzymes, and insulin resistance from baseline; however, no significant difference was observed between the groups. Furthermore, only 19 patients were included in this study. The study authors suggest that no additive benefit of exercise was seen, however conceded that the small number of recruits is a limitation of the study.
Larson-Meyer et $\mathrm{al}^{13}$ compared results from computerized tomography (CT) and MRI to assess the outcome of calorie restriction and exercise on a group of 46 overweight patients. All the patients in this sample were healthy with no underlying liver disease at baseline, and were recruited after 6 months either randomized to calorie restriction alone ( $25 \%$ energy from baseline) or in combination with exercise ( $12.5 \%$ calorie restriction and $12.5 \%$ increase in exercise) or a low calorie diet (15\% weight loss then maintenance) or a control group. The results showed that BMI, insulin resistance, alanine aminotransferase (ALT) levels, and intrahepatic steatosis decrease in all interventional groups (ALT did not decrease in the low calorie diet group). MRI and $\mathrm{CT}$ results correlated closely, but the paper found that MRI was more reliable at imaging lower levels of hepatic fat. This study, although showing interesting results when it comes to imaging, actually may not be relevant as not only did no patients have a diagnosis of NAFLD, but patients were encouraged to drink less than 2 units of alcohol per day, some of whom had been drinking more prior to the trial. Alcohol, therefore, may have altered the results.

Two of the papers we reviewed used US as a measurement of hepatic steatosis improvement over the trial period. Scagiloni et al ${ }^{14}$ carried out a small trial, with twelve participants over a period of 3 months. All participants were encouraged to carry out a diet that had been personally tailored by a dietician, and to increase their physical activity, which was monitored by an armband (comparisons made at baseline and 3 months). Exercise over the 3 month period did not show any significant increase, although there was an average weight loss and BMI decrease of $7.34 \%$. LFTs also did not show any significant improvement but US showed a significant decrease in intrahepatic fat. In contrast, Catalano et $\mathrm{al}^{15}$ used a much larger sample group of 50 over a period of 6 months. All of these participants were encouraged to lose $5 \%$ of their body weight over the trial period, although no specific guidelines were given. At the end of the 6 months, the average weight loss of $57 \%$ of the desired weight loss (5\%) was achieved. Results showed that both BMI and insulin resistance decrease over the trial period and this correlated to a decrease in intrahepatic fat on US. On reviewing blood results, this trial also came to the conclusion that gamma-glutamyl transpeptidase (GGT) levels are the most reliable for assessing parallel modification of serological measurement and morphological changes.

Blood tests are by far the easiest and most accessible way of assessing liver function and this is represented by the number of papers that rely wholly on these as evidence 
for their trials. Six trials in this section used blood results, and not imaging and histology, as a measureable outcome for their trials. Sun et $\mathrm{al}^{16}$ is one of the larger trials included in this review: 1,006 patients were recruited into a 12 month trial where they were randomized to receive lifestyle interventions (NAFLD education including dietary advice and exercise therapy) or to a control group. ALT levels and other markers of metabolic syndrome decreased over the trial period in the lifestyle group (compared to those in the control group), all to a greater extent at 12 months than when reviewed at 6 months. Straznicky et $a{ }^{17}$ recruited 58 patients with three or more markers of metabolic syndrome, rather than being exclusively diagnosed with NAFLD. NAFLD is often described as the hepatic manifestation of metabolic syndrome therefore this paper was included. Over a 12 week period, the 58 patients were randomized to either weight loss by calorie restriction (deficit of 400-900 calories per day), weight loss by calorie restriction and exercise (40 minute bike ride on alternate days), and a control group, a design that is similar to Larson-Meyer et $\mathrm{al}^{13}$ and Shah et al. ${ }^{12}$ The calorie restriction alone group lost on average $7.6 \%$ with a corresponding decrease in ALT of $20 \%$ and GGT of $28 \%$. The calorie restriction and exercise group lost $9.1 \%$ of their baseline weight on average and ALT and GGT levels decreased by $24 \%$ and $33 \%$, respectively. These results showed a significant decrease from baseline but no significant difference between the two groups: they do not support the additive effect of exercise.

Sreenivasa Baba et $\mathrm{al}^{18}$ published another paper using blood tests as its primary follow up. Fifty-nine patients, with US diagnosed NASH, were recruited into a 3-month trial involving an exercise program (45 minutes of exercise five times a week), and a calorie controlled diet for those with a high BMI (25 calories per kg of their ideal body weight). Forty-four patients were compliant to this exercise and diet regime, and those taking part in diet and exercise lost on average $3.1 \mathrm{~kg}$, while those taking part in just the diet lost $0.2 \mathrm{~kg}$, and no significant decrease in weight was seen in the non-compliant group. ALT and aspartate aminotransferase (AST) levels decreased significantly in the compliant group; therefore, this paper draws the conclusion that weight loss is beneficial regardless of exercise participation. This paper included 30 patients who had biopsies at baseline but did not follow these up at the end of the trial. A study by Park et al ${ }^{19}$ had a similar set up to Sreenivasa Baba et al, ${ }^{18}$ though it was a much smaller trial. Twenty-five obese adults with US diagnosed NAFLD were recruited for a 12 month trial. Similar dietary advice of 25-30 calories per $\mathrm{kg}$ of ideal body weight was prescribed, and generalized exercise and behavioral advice was given. Unlike Sreenivasa Baba et al, participants in this trial were considered compliant if they showed a reduction in weight. Again, ALT and AST levels decreased in the compliant group, but in this trial, levels were shown to increase in the non-compliant patients who gained weight.

Kim et $\mathrm{al}^{20}$ used an internet program to encourage participants to improve their diet and increase their exercise. Eighteen men were recruited for 8 weeks to use this program, which set individual goals and targets that could be monitored by a panel. At the end of the trial, participants had significantly increased their exercise but showed no change in dietary habits. Weight and some markers of metabolic syndrome were also shown to decrease, including diastolic blood pressure. Although this paper does not mention NAFLD, as has been previously stated, NAFLD is considered the hepatic manifestation of metabolic syndrome, which is measured. This paper also shows a new innovative way of encouraging a population to lead a healthier lifestyle. The results of this paper therefore contradicts Shah et $\mathrm{al}^{12}$ and Straznicky et al, ${ }^{17}$ which stated that exercise did not play an important part in the improvement of liver steatosis markers, as in this trial exercise does seem to be an important factor.

Venditti et $\mathrm{al}^{21}$ used weight loss to assess whether lifestyle interventions were more effective the first or the second time around. The paper follows the Diabetic Prevention Program ${ }^{22}$ in which participants had been randomized to either 16 sessions of lifestyle interventions, metformin, troflitazone (which was withdrawn due to side effects), or placebo. After this initial trial, all participants were asked if they would continue on a further 6 months of lifestyle interventions and 2,808 agreed. Those in the original lifestyle group were then compared to those who had been in the metformin and placebo groups. Those who had not received lifestyle intervention before were more likely to join this secondary trial and to continue with it, compared to those on their secondary course. Weight loss was therefore greater in the naive group and indeed correlated to the number of sessions attended in this group. Although this paper does not mention NAFLD or liver function it shows the importance of lifestyle intervention leading to weight loss and the problems with compliance.

\section{Diet only regimes}

This section includes the papers using diet as the only intervention; seven papers in total fell under this category. 
Ryan et $\mathrm{al}^{23}$ published a paper in 2006 , which was a post hoc analysis of 52 obese, insulin resistant adults who had taken part in a weight loss program. They had been randomized to receive either a low carbohydrate $\operatorname{diet}(40 \%$ carbohydrate $/ 40 \%$ fat $)$ or a low fat diet $(60 \%$ carbohydrate $/ 25 \%$ fat $)$ for 16 weeks. Both groups lost a significant amount of weight over the trial period but the ALT levels decreased twice as much in those on the low carbohydrate diet compared to the low fat diet. ALT levels were also found to decrease more in those with abnormal levels (30 units/L for men and 19 units/L for women) at baseline than those with normal levels, irrespective of the diet type. Insulin resistance levels were also shown to decrease in both groups with no significant differences between them. The authors draw the conclusion that low carbohydrate diets are more beneficial than low fat diets at reducing ALT levels.

Gasteyger et $\mathrm{al}^{24}$ also used LFTs to assess the effectiveness of dieting. This was again a post hoc analysis with data taken from a trial comparing a low calorie diet with topiramate (an anti-epileptic drug) or placebo. This paper focused on the low calorie diet group, and reviewed the follow up results from 32 and 60 weeks of 147 patients. There was a high drop out in this trial and as a result data were only available for 67 patients at 32 weeks and 68 patients at 60 weeks. On average, patients lost $12.1 \mathrm{~kg}$, men losing more than women over the trial period. AST and ALT levels were shown to decrease in men, while there was a transient increase in women. GGT decreased in men but not in woman and there was no significant change in ALP in either group. These results are interesting, indicating that sex is a key determining factor in change in LFT levels over a trial.

De Luis et $\mathrm{al}^{25}$ reports on a 3-month intervention of hypocaloric diet (either low fat or low carbohydrate) on basic anthropological and biochemical measures in an obese population. An arbitrary definition of NAFLD was made based only on an elevated ALT. After 3 months of dietary intervention, all biochemical parameters improved, along with improvements in BMI and waist circumference. More so, the NAFLD phenotype $(n=30)$ showed improvements in ALT and insulin resistance. Although only small numbers were included in each group, and the NAFLD phenotype was loosely defined, the study reveals that even a short focused intervention (3 months) has an impact on measurable markers associated with metabolic syndrome.

Rodríguez-Hernández et $\mathrm{al}^{26}$ again uses a low fat and low carbohydrate diet to assess their effect on aminotransferase, and this trial is nicely followed up by Haufe et al. ${ }^{27}$ The Rodríguez-Hernández et $\mathrm{al}^{26}$ trial included 54 women, with
US diagnosed NAFLD, and randomized them to a low fat ( $25 \%$ protein, $<10 \%$ fat, $54 \%$ carbohydrate) or low carbohydrate ( $27 \%$ protein, $28 \%$ fat, $45 \%$ carbohydrate) diet for a period of 6 months. At the end of the trial, those on the low carbohydrate diet lost $5.7 \%$ of their body weight and those in the low fat group 5.5\%, a non-significant result. ALT and AST had both decreased in each group but again there was no significant difference. The study by Haufe et $\mathrm{al}^{27}$ follows a similar design but with 102 patients, both male and female, over a 6-month period. In this trial, the diet design was low carbohydrate ( $90 \mathrm{~g}$ of carbohydrate and $0.8 \mathrm{~g}$ protein per $\mathrm{kg}$ weight, $30 \%$ fat) and low fat ( $\leq 20 \%$ fat, $0.8 \mathrm{~g}$ of protein per $\mathrm{kg}$, the remainder carbohydrate). The results were also similar in this trial: a non-significant decrease in weight and ALT between both groups. This study went on to look at intrahepatic fat content by magnetic resonance spectroscopy and tomography. Results showed that intrahepatic fat reduced by $47 \%$ in the low carbohydrate group and $42 \%$ in the low fat group, with no significant difference between the groups. This paper therefore neatly follows from Rodríguez-Hernández et al, with the added evidence of imaging to support the results and a larger sample size.

The small study by Elias et a ${ }^{28}$ recruited 31 participants, with either CT or US diagnosed NAFLD, to carry out a hypocaloric diet (reduction of 500-1,000 calories/day) for 6 months. Seventeen patients were compliant with this diet and ended up losing 5\% of their body weight; with this, they also showed a significant decrease in GGT, ALT, and insulin resistance. At the beginning of the trial, $45 \%$ of the patients had been diagnosed with metabolic syndrome and in the end there were only $10 \%$, all in the non-adherent group. Intrahepatic fat by CT was also shown to decrease in the adherent group compared to the non-adherent group.

Sevastianova et $\mathrm{l}^{29}$ also used imaging to measure the outcome of dieting. This was a small trial involving 16 participants who were required to carry out 3 weeks of over feeding followed by 6 months of a hypocaloric diet. Subjects in this trial were also genotyped to assess the role of genetics in NAFLD. During the 3 week overfeeding liver fat, measured by MRI, increased by $27 \%$ and then fell by $25 \%$ in the hypocaloric phase. ALT levels also increased and then decreased over the trial period. This paper therefore concluded that the changes in liver fat during NAFLD are reversible by dieting. Genetic markers of patatin-like phospholipase domain-containing protein 3 (PNPLA3)-148II and PNPLA3-148MM were investigated in this study. Those with PNPLA3-148II $(\mathrm{n}=7)$ showed a significant increase in lipid fat content while this relationship was not seen in those with 
PNPLA3-148MM ( $\mathrm{n}=9$ ). The study hypothesized the reason behind this change but concluded that the sample size was too small to see a definite relationship.

\section{Exercise only regimes}

Kistler et $\mathrm{al}^{30}$ produced the only paper in this section that used biopsy results as an outcome. The paper was a retrospective study looking at 813 biopsy results of patients with NAFLD and assessed if they were taking part in the recommended guidelines for exercise put forward by the United States Department of Health and Human Services. The guidelines stated that people should be taking part in more than 150 minutes of moderate intensity or 75 minutes of vigorous intensity exercise per week. Participants in this trial were classified as inactive; meeting the moderate intensity guidelines; or meeting the vigorous intensity guidelines. Results of a questionnaire showed that $20 \%$ of the participants met the moderate activity guidelines and $26 \%$ met the vigorous activity guidelines; the majority of patients were inactive. Analysis of the biopsies showed that meeting the vigorous activity guidelines decreases your odds ratio (OR) of having NASH (OR: 0.65); the same was not true for moderate activity. Biopsies also showed that those meeting vigorous activity guidelines had significantly lower odds ratio fibrosis compared to no fibrosis (OR: 0.53); again, the same was not true for moderate activity. Thus this paper concluded that only vigorous activity was enough to cause significant change in liver steatosis and fibrosis.

Hallsworth et $\mathrm{al}^{31}$ constructed a randomized control trial including 19 patients with MRI diagnosed NAFLD. The patients were randomized to take part in 45-60 minutes of resistance exercise three times a week for 8 weeks, or be in a control group. They were only included in the final results if they lost less than $5 \%$ of their body weight, therefore excluding the weight loss from affecting the results. Participants were required to wear armbands 7 days prior to the intervention and for 7 days afterwards to assess the change. Results showed that there was a $13 \%$ decrease in intrahepatic fat, recorded by MRI, in the intervention group with no change in the control group. There was improvement in insulin resistance but no change in ALT or blood lipid levels. Similar to Hallsworth et al, ${ }^{31}$ Sullivan et $\mathrm{al}^{32}$ also recruited a small sample, 18 participants with NAFLD, to participate in a randomized control trial. Although in this study, the intervention group was required to carry out 30-60 minutes of exercise five times a week, one of these was under direct supervision. Again, similarly, MRI scans were used to determine the intrahepatic fat content, which showed a mild decrease in the intervention group compared to the control. The same was also true for ALT values: the decrease in ALT correlated with the decrease in intrahepatic fat in the intervention group. These trials have a similar design and they also both have a small sample size that may have led to bias.

Fealy et $\mathrm{al}^{33}$ carried out a small short lived trial to assess the effect of exercise training on markers of hepatocyte apoptosis. Thirteen patients were recruited into the trial, where they were required to carry out 60 minutes of aerobic activity at $80 \%-85 \%$ of their maximum heart rate for seven consecutive days. MRI scans were performed on all participants pre and post-trial and these showed no change in intrahepatic fat during the intervention. Blood tests did show some changes, those with raised ALT at baseline had decreased levels after the trial, and blood glucose levels, and insulin sensitivity also showed improvements. This trial was very short lived which may explain the limited change in markers of NAFLD.

The effect of a short prescribed program of endurance exercise was assessed for improvements in liver fat content in a cohort including lean and obese individuals by Tarnopolsky et al. ${ }^{34}$ The study design precluded weight loss as an end point, instead focusing on participation in a moderate intensity program of endurance exercise (stationary bike cycling) and assessing its effect on liver fat content principally. After a 3-month period of endurance exercise, improvements in fitness (maximal oxygen consumption) and reduction in waist circumference were noted in both the lean and obese groups, regardless of sex. No change in BMI was seen. Hepatic lipid content, as measured by CT (liver attenuation), did not change post exercise, regardless of group or sex. Furthermore, no improvement in liver enzymes or insulin resistance was observed. This study is limited by the small numbers of patients included and by the apparent liver health of the group the obese men had a pre-exercise ALT of $30.3 \pm 4.8 \mathrm{U} / 1$ and the obese women 16.8 $\pm 2.2 \mathrm{U} / 1$. Furthermore, the intervention period is short at 3 months. No measurement of lipid profile was discussed; early changes in triglyceride content may have supported and encouraged participation in a longer program. This study does however highlight the need for intervention programs designed to induce weight loss. This study was deliberately designed not to induce weight loss. With no effect on liver fat content, this supports the notion that loss of body weight is important for improving liver health in obese individuals.

Bae et $\mathrm{al}^{35}$ is one of the larger trials reviewed in this discussion, with a cross-sectional analysis taken on 72,359 Korean adults. Baseline results were collected from these patients at the Kangbuk Samsung Hospital Total 
Healthcare Centre, and the diagnosis of NAFLD was made by US. The participants were asked to fill out a questionnaire recording how much exercise they carried out on a weekly basis; regular exercise was defined as 30 minutes more than three times a week for three consecutive months. Results showed that 12,967 patients fitted the criteria of carrying out regular exercise. There were 19,921 patients with NAFLD and this number was significantly higher in those who did not exercise regularly. The same was true for BMI: NAFLD occurrence was higher with those with a greater BMI and liver function levels were lower on average if the patient took part in regular exercise.

St George et $\mathrm{al}^{36,37}$ carried out two papers that are included in this review. Both have a similar design with a low intensity lifestyle intervention group (three counseling sessions throughout the trial), moderate intensity lifestyle intervention group (six counseling sessions throughout the trial), a long term group (receiving the same initial intervention as the moderate group but with further sessions up to 12 months), and a control group. All participants were encouraged to carry out the same diet with a reduction in energy intake of 1700-2400 kJ over a 3 month period and to increase their exercise to 150-200 minutes a week. The St George et al study that was published in October $2008^{37}$ included 152 participants followed up for a 9-month period. Exercise was shown to be significantly increased in both the low and moderate intensity exercise encouragement groups, with no change in the control group. Weight loss was found to be highest in those receiving moderate intensity lifestyle interventions and lowest in the control group; liver function tests were also found to correlate with weight loss. The moderate intensity group was also shown to have decreased markers of metabolic risk factors at the end of the trial period. The study by St George et al published in $2009^{36}$ categorized 141 patients into whether they increased their physical activity by more than 60 minutes per week, maintained their physical activity, or decreased their physical activity. In the moderate intensity encouragement group $64 \%$ and $63 \%$ from the low intensity encouragement group increased their activity from base line. This was a significant difference from the control group with only $16 \%$ increasing their physical exercise. The greatest changes were in insulin resistance, metabolic risk factors, and LFTs seen in those who increased their physical activity.

Gerber et $\mathrm{al}^{38}$ carried out a cross-sectional analysis of a population using data from two surveys carried out between 2003-2004 and 2005-2006 in the United States. The data from these surveys were mostly self-reported, collecting baseline characteristics, lifestyle choices, and medical backgrounds of the subjects. Diagnosis of NAFLD was made using the Fatty Liver Index, ${ }^{39}$ a simple scoring system that predicts the degree of hepatosteatosis using the baseline characteristics of the subjects. Patients were asked to wear activity monitor arm bands, similar to the ones used in Hallsworth et al, ${ }^{31}$ for seven consecutive days. Those with NAFLD were found to be less likely to take part in regular exercise, and having diabetes as well, made this relationship stronger. Results also showed that exercise did not correlate with aminotransferase levels. Although this paper had a large sample size and an accurate way of recording exercise in participants, the trial period was very short, which may not reflect a true representation of the participants' exercise levels.

\section{Pharmacological interventions used concomitantly with or in comparison to lifestyle interventions}

There are many theories about various medications that may improve the outcome of NAFLD; this review paper includes three trials using a variety of medications.

Twenty-three women were recruited by Méndez-Sánchez et al, ${ }^{40}$ for a double blinded control trial testing the effects of ursodeoxycholic acid on patients with NAFLD. Patients were randomized to receive a healthy diet $(1200 \mathrm{kcal} /$ day of which $20 \%$ was fat, $60 \%$ carbohydrate, and $20 \%$ protein) with either ursodeoxycholic acid or placebo. Both groups lost weight over the trial with no significant difference between the two groups. ALT decreased more in those taking ursodeoxycholic acid compared to those who were not, but other than that there was no significantly different blood results between the two groups. US was performed on the patients at the beginning and end of the trial, and fat liver content was shown to decrease in both groups but again no significant difference between the two groups was found. This study therefore concludes that weight loss is the most important influence on NAFLD.

Viusid (Catalysis, S.L., Madrid, Spain), an antioxidant nutritional supplement, was investigated by Vilar Gomez et $\mathrm{al}^{41}$ in a randomized control trial. Sixty patients, with biopsy proven NAFLD, were randomized to receive a hypocaloric diet and exercise with or without Viusid. Monitoring of the diet and exercise was done by self-reporting and biopsies were taken at baseline and at the end of the 24 week trial, which were examined by a pathologist. There were eleven dropouts over the trial for various reasons, and Viusid was reported to have side effects including nausea and diarrhea in some cases. ALT and AST decreased significantly over the trial with no significant difference between 
the two trial groups. The Viusid group did show a significant improvement in liver histology, and improvements in fibrosis and steatosis, as did the control group. Between group comparison showed significant improvements in the Viusid group in all parameters of the NAS score (but not the fibrosis grade) compared to the control. There was a relatively high dropout rate in this trial, and those who dropped out were not accurately followed up. The reason behind these dropouts were not reported; these would have been interesting as they may have impacted on Viusid's use in a clinical setting.

Krakoff et $\mathrm{al}^{42}$ used data from the Diabetic Prevention Program, similar to Venditti et al. ${ }^{21}$ Venditti et al focused on the placebo group and followed them up after the trial, whereas Krakoff et al compared a metformin group to a placebo group. In Krakoff et al both groups received diet and exercise programs to induce weight loss, and the 2,153 had also been randomized to receive either placebo or metformin for an average trial period of 3.2 years. ALT levels rose in both groups over the trial, although the rise was greatest in the placebo group. The study also showed that the incidence of abnormal ALT decreased with increasing amount of weight loss. All participants in this trial had normal ALT at baseline and NAFLD status was not known, which may explain why these results do not follow the same trends as other papers.

Akcam et $\mathrm{al}^{43}$ performed a small randomized double blind parallel group clinical trial assessing the effect of diet and exercise alone or in combination with either metformin or vitamin $\mathrm{E}$ on various markers of NAFLD, including ultrasonographically assessed liver steatosis, insulin resistance, lipid profile, and BMI. They recruited 67 adolescents with US defined NAFLD to three groups all of whom received personalized and prescribed diet and exercise regimes. One group also received metformin ( $850 \mathrm{mg}$ once daily) while another group received vitamin E (400 U daily). Patients were assessed and followed up for 6 months. BMI improved in all groups, more so in the metformin and vitamin $\mathrm{E}$ groups, although this did not achieve statistical significance. Unsurprisingly, insulin resistance was most improved in the metformin treated group, with only slight improvement seen in the other two groups. No direct markers of oxidative stress improvement were measured, although tumor necrosis factor (TNF)-alpha did not improve significantly in any of the three groups. At the conclusion of the study period, repeat liver US was performed, with the metformin group showing the greatest improvement - almost double that of the diet and exercise alone group and the vitamin E group. This well designed study compared pharmacological intervention with either metformin or vitamin $\mathrm{E}$ with diet and exercise alone.
The short duration of the study is a limitation; perhaps greater effects from all three interventions would be more apparent if the study period was extended. However, although the authors highlight the improvements seen with metformin in particular, the diet and exercise alone group did achieve an improvement in BMI supporting the role of this fundamental intervention in a young cohort.

\section{Conclusion}

Overall, 34 studies were reviewed in this paper, 14 using diet and exercise interventions, seven with just diet interventions, nine with just exercise intervention, and four using medications as interventions.

All of the 14 studies using diet and exercise as interventions showed improvements in their various measured outcomes. Although there were a few, including Shah et $\mathrm{al}^{12}$ and Straznicky et al, ${ }^{17}$ who showed that exercise had no additive effect when used in conjunction with diet to improve liver steatosis markers. This was contradicted by Kim et $\mathrm{al}^{20}$ and, indeed, the majority of the studies using just exercise interventions showed that exercise does improve liver markers of steatosis.

Of those in the exclusively exercise interventions all but one study showed a relationship between increased exercise and improvement of hepatic markers. Tarnopolsky et al ${ }^{34}$ showed no improvement in hepatic fat content and blood results with increased exercise. This was concluded to be due to the relatively small sample size and short trial period, as well as participants being "healthy" at recruitment. Fealy et $\mathrm{al}^{33}$ also showed no improvement in hepatic steatosis on US but did show improvement in blood results when participants increased their exercise. Three of the studies looked at the comparison between different intensities of exercise, both St George et $\mathrm{al}^{36,37}$ studies showed improvements with moderate intensity exercise while Kistler et $\mathrm{al}^{30}$ only showed improvements with vigorous activity.

Two of the studies were cross-sectional analyses, Bae et $\mathrm{al}^{35}$ and Gerber et $\mathrm{al},{ }^{38}$ both of these showed a strong relationship between the proportion of the population with NAFLD and the low level of activity that they took part in.

Seven studies used dietary interventions, all of which showed improvements in hepatic steatosis markers. Two of the trials compared low carbohydrate and low fat diets, Rodríguez-Hernández et $\mathrm{al}^{26}$ showed similar results between the two diets whereas Ryan et $\mathrm{al}^{23}$ showed better results with those on the low carbohydrate diet.

In the medication intervention group there were four studies: Méndez-Sánchez et $\mathrm{al}^{40}$ using ursodeoxycholic acid, 
Vilar Gomez et $\mathrm{al}^{41}$ using Viusid (an antioxidant nutritional supplement), Krakoff et al ${ }^{42}$ using metformin, and Akcam et $\mathrm{al}^{43}$ compared metformin and vitamin $\mathrm{E}$. All the studies apart from the one by Vilar Gomez et $\mathrm{al}^{41}$ concluded that there was no particular benefit from their intervention and that weight loss by diet and exercise was the most important factor. The Vilar Gomez et al study ${ }^{41}$ using Viusid did show improvement, but there were issues with side effects from the drug which require further investigation before it can be endorsed.

All papers used a variety of follow up methods, including biopsy, imaging and liver function tests. Four studies used biopsies for follow up, three in the diet and exercise section, and one in the exercise section. Biopsy results are considered the gold standard for staging and grading of NAFLD. There were also different imaging methods used, including MRI, CT, and US. Larson-Meyer et $\mathrm{al}^{13}$ compared CT and MRI results, concluding that MRI produced better results with respect to lower levels of hepatic fat content.

Practice guidelines set forth by key stakeholders in NAFLD published in 2012 endorse the vital role of lifestyle intervention in the management of NAFLD. ${ }^{44}$ Weight loss either by hypocaloric diet or in combination with increased physical activity to attain a $3 \%-5 \%$ loss in body weight is required to improved steatosis, with up to $10 \%$ loss of body weight required to improve necroinflammation.

This review concludes that lifestyle intervention is a vital and central component to the treatment of NAFLD and metabolic syndrome. The optimal strategy is not yet realized, although the importance of weight loss and exercise is paramount. Sustainability of any intervention is key to success; however, it is an elusive end point.

\section{Disclosure}

The authors report no conflicts of interest in this work.

\section{References}

1. Neuschwander-Tetri BA, Caldwell SH. Nonalcoholic steatohepatitis: summary of an AASLD Single Topic Conference. Hepatology. 2003;37(5):1202-1219.

2. Adams L, Angulo P. Treatment of non-acoholic fatty liver disease. Postgraduate Medical Journal. 2006;82(967):315-322.

3. Bellentani S, Scaglioni F, Marino M, Bedogni G. Epidemiology of nonalcoholic fatty liver disease. Dig Dis. 2010;28(1):155-161.

4. Tolman KG, Dalpiaz AS. Treatment of non-alcoholic fatty liver disease. Ther Clin Risk Manag. 2007;3(6):1153-1163.

5. Bedogni G, Miglioli L, Masutti F, Tiribelli C, Marchesini G, Bellentani S. Prevalence of and risk factors for nonalcoholic fatty liver disease: the Dionysos nutrition and liver study. Hepatology. 2005;42(1):44-52.

6. Newsome PN, Allison ME, Andrews PA, et al; British Transplant Society. Guidelines for liver transplantation for patients with non-alcoholic steatohepatitis. Gut. 2012;61(4):484-500.

7. James WP. The epidemiology of obesity: the size of the problem. J Intern Med. 2008;263(4):336-352.
8. Promrat K, Kleiner DE, Niemeier HM, et al. Randomized controlled trial testing the effects of weight loss on nonalcoholic steatohepatitis. Hepatology. 2010;51(1):121-129.

9. Jin YJ, Kim KM, Hwang S, et al. Exercise and diet modification in non-obese non-alcoholic fatty liver disease: analysis of biopsies of living liver donors. J Gastroenterol Hepatol. 2012;27(8): 1341-1347.

10. Bhat G, Baba CS, Pandey A, Kumari N, Choudhuri G. Life style modification improves insulin resistance and liver histology in patients with non-alcoholic fatty liver disease. World J Hepatol. 2012;4(7): 209-217.

11. Lazo M, Solga SF, Horska A, et al; Fatty Liver Subgroup of the Look AHEAD Research Group. Effect of a 12-month intensive lifestyle intervention on hepatic steatosis in adults with type 2 diabetes. Diabetes Care. 2010;33(10):2156-2163.

12. Shah K, Stufflebam A, Hilton TN, Sinacore DR, Klein S, Villareal DT. Diet and exercise interventions reduce intrahepatic fat content and improve insulin sensitivity in obese older adults. Obesity (Silver Spring). 2009; 17(12):2162-2168.

13. Larson-Meyer DE, Newcomer BR, Heilbronn LK, et al; Pennington CALERIE Team. Effect of 6-month calorie restriction and exercise on serum and liver lipids and markers of liver function. Obesity (Silver Spring). 2008;16(6):1355-1362.

14. Scaglioni F, Marino M, Ciccia S, et al. Short-term multidisciplinary non-pharmacological intervention is effective in reducing liver fat content assessed non-invasively in patients with nonalcoholic fatty liver disease (NAFLD). Clin Res Hepatol Gastroenterol. 2013;37(4): 353-358.

15. Catalano D, Trovato GM, Martines GF, Randazzo M, Tonzuso A. Bright liver, body composition and insulin resistance changes with nutritional intervention: a follow-up study. Liver Int. 2008;28(9):1280-1287.

16. Sun WH, Song MQ, Jiang CQ, et al. Lifestyle intervention in non-alcoholic fatty liver disease in Chengyang District, Qingdao, China. World J Hepatol. 2012;4(7):224-230.

17. Straznicky NE, Lambert EA, Grima MT, et al. The effects of dietary weight loss with or without exercise training on liver enzymes in obese metabolic syndrome subjects. Diabetes Obes Metab. 2012;14(2): $139-148$.

18. Sreenivasa Baba C, Alexander G, Kalyani B, et al. Effect of exercise and dietary modification on serum aminotransferase levels in patients with nonalcoholic steatohepatitis. J Gastroenterol Hepatol. 2006; 21(1 Pt 1):191-198.

19. Park HS, Kim MW, Shin ES. Effect of weight control on hepatic abnormalities in obese patients with fatty liver. J Korean Med Sci. 1995;10(6):414-421.

20. Kim CJ, Kang S. Development and a pilot test of an internet-based cardiovascular risk reduction program for Korean male workers with metabolic syndrome. Comput Inform Nurs. 2013;31(4):157-166.

21. Venditti EM, Bray GA, Carrion-Petersen ML, et al; Diabetes Prevention Program Research Group. First versus repeat treatment with a lifestyle intervention program: attendance and weight loss outcomes. Int J Obes (Lond). 2008;32(10):1537-1544.

22. Knowler WC, Barrett-Connor E, Fowler SE, et al. Diabetes Prevention Program Research Group. Reduction in the incidence of type 2 diabetes with lifestyle intervention or metformin. New England Journal Med. 2002; 346:393-403.

23. Ryan MC, Abbasi F, Lamendola C, Carter S, McLaughlin TL. Serum alanine aminotransferase levels decrease further with carbohydrate than fat restriction in insulin-resistant adults. Diabetes Care. 2007;30(5): $1075-1080$.

24. Gasteyger C, Larsen TM, Vercruysse F, Astrup A. Effect of a dietaryinduced weight loss on liver enzymes in obese subjects. Am J Clin Nutr. 2008;87(5):1141-1147.

25. de Luis DA, Aller R, Izaola O, Gonzalez Sagrado M, Conde R. Effect of two different hypocaloric diets in transaminases and insulin resistance in nonalcoholic fatty liver disease and obese patients. Nutr Hosp. 2010;25(5):730-735. 
26. Rodríguez-Hernández H, Cervantes-Huerta M, Rodríguez-Moran M, Guerrero-Romero F. Decrease of aminotransferase levels in obese women is related to body weight reduction, irrespective of type of diet. Ann Hepatol. 2011;10(4):486-492.

27. Haufe S, Engeli S, Kast P, et al. Randomized comparison of reduced fat and reduced carbohydrate hypocaloric diets on intrahepatic fat in overweight and obese human subjects. Hepatology. 2011;53(5): $1504-1514$.

28. Elias MC, Parise ER, de Carvalho L, Szejnfeld D, Netto JP. Effect of 6-month nutritional intervention on non-alcoholic fatty liver disease. Nutrition. 2010;26(11-12):1094-1099.

29. Sevastianova K, Santos A, Kotronen A, et al. Effect of short-term carbohydrate overfeeding and long-term weight loss on liver fat in overweight humans. Am J Clin Nutr. 2012;96(4):727-734.

30. Kistler KD, Brunt EM, Clark JM, Diehl AM, Sallis JF, Schwimmer JB; NASH CRN Research Group. Physical activity recommendations, exercise intensity, and histological severity of nonalcoholic fatty liver disease. Am J Gastroenterol. 2011;106(3):460-468; quiz 469.

31. Hallsworth K, Fattakhova G, Hollingsworth KG, et al. Resistance exercise reduces liver fat and its mediators in non-alcoholic fatty liver disease independent of weight loss. Gut. 2011;60(9):1278-1283.

32. Sullivan S, Kirk EP, Mittendorfer B, Patterson BW, Klein S. Randomized trial of exercise effect on intrahepatic triglyceride content and lipid kinetics in nonalcoholic fatty liver disease. Hepatology. 2012;55(6):1738-1745.

33. Fealy CE, Haus JM, Solomon TP, et al. Short-term exercise reduces markers of hepatocyte apoptosis in nonalcoholic fatty liver disease. J Appl Physiol (1985). 2012;113(1):1-6.

34. Tarnopolsky MA, Rennie CD, Robertshaw HA, Fedak-Tarnopolsky SN, Devries MC, Hamadeh MJ. Influence of endurance exercise training and sex on intramyocellular lipid and mitochondrial ultrastructure, substrate use, and mitochondrial enzyme activity. Am J Physiol Regul Integr Comp Physiol. 2007;292(3):R1271-R1278.

35. Bae JC, Suh S, Park SE, et al. Regular exercise is associated with a reduction in the risk of NAFLD and decreased liver enzymes in individuals with NAFLD independent of obesity in Korean adults. PLoS One. 2012;7(10):e46819.
36. St George A, Bauman A, Johnston A, Farrell G, Chey T, George J. Independent effects of physical activity in patients with nonalcoholic fatty liver disease. Hepatology. 2009;50(1):68-76.

37. St George A, Bauman A, Johnston A, Farrell G, Chey T, George J. Effect of a lifestyle intervention in patients with abnormal liver enzymes and metabolic risk factors. J Gastroenterol Hepatol. 2009;24(3): 399-407.

38. Gerber L, Otgonsuren M, Mishra A, et al. Non-alcoholic fatty liver disease (NAFLD) is associated with low level of physical activity: a population-based study. Aliment Pharmacol Ther. 2012;36(8): 772-781.

39. Bedogni G, Bellentani S, Miglioli L, et al. The Fatty Liver Index: a simple and accurate predictor of hepatic steatosis in the general population. BMC Gastroenterol. 2006; 6: 33.

40. Méndez-Sánchez N, González V, Chávez-Tapia N et al,. Weight reduction and ursodeoxycholic acid in subjects with non alcoholic fatty liver disease. A double blind placebo controlled trial. Ann Hepatol. 2004;3(3):108-112.

41. Vilar Gomez E, Rodriguez De Miranda A, Gra Oramas B, et al. Clinical trial: a nutritional supplement Viusid, in combination with diet and exercise, in patients with nonalcoholic fatty liver disease. Aliment Pharmacol Ther. 2009;30(10):999-1009.

42. Krakoff J, Clark JM, Crandall JP, et al; Diabetes Prevention Program Research Group. Effects of metformin and weight loss on serum alanine aminotransferase activity in the diabetes prevention program. Obesity (Silver Spring). 2010;18(9):1762-1767.

43. Akcam M, Boyaci A, Pirgon O, Kaya S, Uysal S, Dundar BN. Therapeutic effect of metformin and vitamin $E$ versus prescriptive diet in obese adolescents with fatty liver. Int J Vitam Nutr Res. 2011;81(6): 398-406.

44. Chalasani N, Younossi Z, Lavine JE, et al. The diagnosis and management of non-alcoholic fatty liver disease: practice Guideline by the American Association for the Study of Liver Diseases, American College of Gastroenterology, and the American Gastroenterological Association. Hepatology. 2012;55(6):2005-2023.
Hepatic Medicine: Evidence and Research

\section{Publish your work in this journal}

Hepatic Medicine: Evidence and Research is an international, peerreviewed, open access journal covering all aspects of adult and pediatric hepatology in the clinic and laboratory including the following topics: Pathology, pathophysiology of hepatic disease; Investigation and treatment of hepatic disease; Pharmacology of drugs used for

\section{Dovepress}

the treatment of hepatic disease. Issues of patient safety and quality of care will also be considered. The manuscript management system is completely online and includes a very quick and fair peer-review system, which is all easy to use. Visit http://www.dovepress.com/ testimonials.php to read real quotes from published authors. 